\title{
Single-marker and multi-marker approaches to appraise the relationships between biomarkers and microalbuminuria in Chinese middle-aged and elderly from communities: a cross-sectional analysis
}

Shihui $\mathrm{Fu}^{1,2+}, \mathrm{Yi} \mathrm{GuO}^{2 \dagger}$, Zhao Zhang ${ }^{2}$, Leiming Luo ${ }^{1 *}$ and Ping Ye 1* $^{*}$

\begin{abstract}
Background: Analyzing the relationships between biomarkers representing distinct pathophysiologic pathways and microalbuminuria (MA) can strengthen the identifying ability for renal damage and illuminate previously unrecognized pathways for the pathogenesis of renal damage. The current analysis was to clarify the associations between biomarkers, including N-terminal prohormone of brain natriuretic peptide (NT-proBNP), high-sensitivity C-reactive protein (hsCRP), homocysteine and uric acid (UA), and MA in Chinese middle-aged and elderly from communities.
\end{abstract}

Methods: All 839 residents had complete set of these biomarkers and full assessment of MA.

Results: Prevalence of participants with MA was 13.5\% (113 participants). Levels of age, systolic blood pressure (SBP), fasting blood glucose (FBG), homocysteine and NT-proBNP and proportion of cigarette smoking in participants with MA significantly exceeded those in participants without MA ( $p<0.05$ for all). In single-marker and multi-marker models of linear and logistic regression analyses, homocysteine and NT-proBNP levels ( $p<0.05$ for all) rather than hsCRP and UA levels ( $p>0.05$ for all) were statistically significant in relation to MA. Additionally, no matter which biomarker was directed at, levels of age, SBP and FBG and proportion of cigarette smoking had significant associations with MA. Homocysteine and NT-proBNP levels ( $p<0.05$ for all) rather than hsCRP and UA levels ( $p>0.05$ for all) had significant abilities to identify MA.

Conclusion: Both single-marker and multi-marker analyses confirmed that homocysteine and NT-proBNP were associated with MA in Chinese middle-aged and elderly from communities after adjustment for multiple confounders.

Keywords: Biomarkers, Communities, Microalbuminuria, Middle-aged and elderly

\section{Background}

Renal damage has significant associations with stroke, cardiovascular disease, peripheral vascular disease and all-cause mortality [1-3]. Microalbuminuria (MA) is well known as an early sign of kidney damage, and early screening of MA is significant to identify renal damage [4]. Analyzing the relationships between MA and biomarkers

\footnotetext{
*Correspondence: Ileim@sina.com; sci301@126.com

${ }^{\dagger}$ Equal contributors

'Department of Geriatric Cardiology, Chinese People's Liberation Army General Hospital, Beijing, China

Full list of author information is available at the end of the article
}

representing distinct biologic can strengthen our identifying ability for renal damage at early stage [5]. Previous studies relating biomarkers to MA have conducted mainly among diabetic and hypertensive individuals, and most of their samples were white, limiting the generalizability to other ethnicities [6-8]. Therefore, scarce studies have examined this kind of relationships in Chinese community population. The current analysis aimed to clarify the associations between biomarkers and MA in Chinese middle-aged and elderly from communities. What is more, the current analysis constructed single-marker and multi-marker models of linear and logistic regression analyses simultaneously

(c) The Author(s). 2018 Open Access This article is distributed under the terms of the Creative Commons Attribution 4.0 International License (http://creativecommons.org/licenses/by/4.0/), which permits unrestricted use, distribution, and 
and tried to test whether these associations were independent out of multiple potential confounders.

\section{Methods \\ Study population}

For the current analysis, 841 participants aged 45 years and over were included from a large health check-up survey, which began in May 2007 and ended in July 2009. A stratified cluster sampling was applied, and three districts (Fengtai, Shijingshan and Daxing) were chosen from eighteen districts in Beijing. Four communities were then chosen from these districts, and participants were finally chosen from these communities. After 2 participants with macroalbuminuria excluded, 839 participants attended the final analysis. All participants in the current analysis had no missing values for any covariates.

\section{Physical examination}

Physical examination was performed by full trained physicians. Height and weight were measured, and body mass index (BMI) was defined as weight $(\mathrm{kg})$ divided by height $(\mathrm{m})$ squared. Participants with systolic blood pressure $(\mathrm{SBP}) \geq 140 \mathrm{mmHg}$, diastolic blood pressure $(\mathrm{DBP}) \geq$ $90 \mathrm{mmHg}$ or hypotensive drugs were regarded to have hypertension. Mercury sphygmomanometer was applied to measure blood pressure twice and obtain an average value (Yuwell medical equipment \& supply Co., Ltd., Jiangsu, China). Two measures are significantly related to each other (SBP: $r=0.925, p<0.001$; DBP: $r=0.908, p<0.001$ ). Cigarette smoking was defined as the consumption of one or more cigarettes per day for at least one recent year.

\section{Laboratory test}

Fasting blood specimen was routinely collected between 8:00 AM and 10:00 AM, and stored at $4{ }^{\circ} \mathrm{C}$ until analyzed by the central laboratory on the same day. $\mathrm{N}$-terminal prohormone of brain natriuretic peptide (NT-proBNP) (representing natriuretic peptide system; measured by electrochemiluminescence immunoassays [Roche Products Ltd., Basel, Switzerland] with an autoanalyzer [COBAS 6000; Roche Products Ltd., Basel, Switzerland]); highsensitivity C-reactive protein (hsCRP) (representing systemic inflammation; measured by immunoturbidimetric assays with a Dimension RxL Max analyzer [Siemens Healthcare Diagnostics Inc., Munich, Germany]); homocysteine (representing oxidative stress; measured by highperformance chromatography with fluorometric detection); and uric acid (UA) (representing nucleic acid metabolism; measured by enzymatic assays [Roche Products Ltd., Basel, Switzerland] with an autoanalyzer [COBAS 6000; Roche Products Ltd., Basel, Switzerland]). Additionally, concentrations of fasting blood glucose (FBG), triglyceride (TG), high-density lipoprotein cholesterol (HDL-c) and lowdensity lipoprotein cholesterol (LDL-c) were quantified by enzymatic assays (Roche Products Ltd., Basel, Switzerland) with an autoanalyzer (Roche Products Ltd., Basel, Switzerland). Type 2 diabetes referred to those with FBG $\geq$ $7.0 \mathrm{mmol} / \mathrm{L}$ or hypoglycemic treatment. Concentrations of serum creatinine were measured by enzymatic assays (Roche Products Ltd., Basel, Switzerland) with a Hitachi 7600 autoanalyzer (Hitachi, Tokyo, Japan). Estimated glomerular filtration rate (eGFR) was calculated with Chinese modified Modification of Diet in Renal Disease equation: $175 \times$ serum creatinine $(\mathrm{mg} / \mathrm{dl})^{-1.234} \times$ age $(\text { year })^{-0.179} \times 0.79$ (if female) [9]. Urinary albumin (UMA) was measured on at least $5 \mathrm{~mL}$ of midstream urine from morning urine. Participants were categorized as those without MA and macroalbuminuria $(<20 \mathrm{mg} / \mathrm{L})$, those with MA $(20-199 \mathrm{mg} / \mathrm{L})$ and those with macroalbuminuria $(\geq$ $200 \mathrm{mg} / \mathrm{L})$.

\section{Statistical analysis}

Statistical analysis was undertaken by Statistical Package for Social Science version 17 software (SPSS Inc., Chicago, IL, USA). Categorical variable was described with number and proportion. Continuous variable (normal distribution) was described with mean and standard deviation. Continuous variable (skewed distribution) was described with median and interquartile range. Student's t-test (continuous variable with normal distribution), Mann-Whitney U test (continuous variable with skewed distribution) and Chi-squared test (categorical variable) were performed to compare differences between participants with and without MA. In order to analyze the independent relationships between biomarkers and MA, essential variable was log-transformed in accordance with multinormality assumption, and multiple linear [independent variable: biomarkers; dependent variable: UMA] and logistic regression models (independent variable: biomarkers; dependent variable: MA) were evaluated following three steps: 1) step 1 included single biomarker in the model (covariates: age and gender); 2) step 2 included single biomarker in the model (covariates: age, gender, BMI, cigarette smoking, SBP, triglyceride, HDL-c, LDL-c, FBG and eGFR); and 3) step 3 included all biomarkers simultaneously in the model (covariates: age, gender, BMI, cigarette smoking, SBP, triglyceride, HDLc, LDL-c, FBG and eGFR). Statistical significance was deemed as two-tailed $P<0.05$.

\section{Results}

\section{Baseline characteristics}

Age ranged from 48 to 89 years, with a median of 67 years; $34.4 \%$ were males, $25.3 \%$ had cigarette smoking, $59.7 \%$ had hypertension, and $25.0 \%$ had type 2 diabetes. Prevalence of participants with MA was 13.5\% (113 participants). Table 1 compared the characteristics of study population according to presence versus absence of MA, and levels of age, BMI, SBP, DBP, FBG, homocysteine and NT-proBNP 
Table 1 Characteristics of study population

\begin{tabular}{|c|c|c|c|c|}
\hline Characteristics & $\begin{array}{l}\text { ALL } \\
(n=839)\end{array}$ & $\begin{array}{l}\text { NA } \\
(n=726)\end{array}$ & $\begin{array}{l}\mathrm{MA} \\
(n=113)\end{array}$ & $P$ value \\
\hline Age (year) & $67(61-72)$ & $62(56-69)$ & $67(62-72)$ & $<0.001$ \\
\hline Males (\%) & $289(34.4)$ & $246(33.9)$ & $43(38.1)$ & 0.386 \\
\hline BMI $\left(\mathrm{kg} / \mathrm{m}^{2}\right)$ & $25.26(23.11-27.64)$ & $25.11(22.96-27.62)$ & $26.22(24.29-27.70)$ & 0.009 \\
\hline Cigarette smoking (\%) & $212(25.3)$ & $169(23.3)$ & $43(38.1)$ & 0.001 \\
\hline Hypertension (\%) & $501(59.7)$ & $417(57.4)$ & $84(74.3)$ & 0.001 \\
\hline Type 2 diabetes (\%) & $210(25.0)$ & $182(25.1)$ & $28(24.8)$ & 0.947 \\
\hline SBP $(\mathrm{mmHg})$ & $130(120-144)$ & $130(120-142)$ & $138(123-157)$ & $<0.001$ \\
\hline $\mathrm{DBP}(\mathrm{mmHg})$ & 77 (70-83) & $75(70-81)$ & $80(75-90)$ & $<0.001$ \\
\hline Triglyceride (mmol/L) & $1.53(1.16-2.15)$ & $1.53(1.15-2.12)$ & $1.54(1.21-2.40)$ & 0.350 \\
\hline $\mathrm{HDL}-\mathrm{c}(\mathrm{mmol} / \mathrm{L})$ & $1.34(1.10-1.59)$ & $1.33(1.09-1.60)$ & $1.36(1.15-1.54)$ & 0.695 \\
\hline LDL-c (mmol/L) & $3.01(2.55-3.43)$ & $3.02(2.56-3.42)$ & $2.95(2.52-3.48)$ & 0.803 \\
\hline FBG $(\mathrm{mmol} / \mathrm{L})$ & $5.01(4.35-5.79)$ & $4.88(4.30-5.70)$ & $5.48(4.92-6.39)$ & $<0.001$ \\
\hline eGFR $\left(\mathrm{ml} / \mathrm{min} / 1.73 \mathrm{~m}^{2}\right)$ & 86.82 (78.09-97.65) & 86.61 (78.13-97.57) & 89.09 (77.54-98.47) & 0.972 \\
\hline UMA (mg/L) & $1.39(0.61-6.35)$ & $1.12(0.55-2.93)$ & 41.04 (28.18-94.35) & $<0.001$ \\
\hline UA $(\mu \mathrm{mol} / \mathrm{L})$ & $288.30(241.80-343.00)$ & $288.60(241.30-341.28)$ & $286.10(244.65-348.05)$ & 0.455 \\
\hline Homocysteine $(\mu \mathrm{mol} / \mathrm{L})$ & $18.30(15.30-22.40)$ & $17.90(15.10-21.63)$ & $20.70(16.85-24.35)$ & $<0.001$ \\
\hline $\mathrm{HsCRP}(\mathrm{mg} / \mathrm{dl})$ & $0.21(0.13-0.35)$ & $0.21(0.13-0.35)$ & $0.21(0.11-0.34)$ & 0.107 \\
\hline NT-proBNP (pg/mL) & $47.60(23.20-90.00)$ & $45.20(21.75-85.90)$ & 73.75 (36.94-132.10) & $<0.001$ \\
\hline ACEl/ARBs (\%) & $34(4.1)$ & $23(3.2)$ & $11(9.7)$ & 0.002 \\
\hline Statins (\%) & $32(3.8)$ & $31(4.3)$ & $1(0.9)$ & 0.138 \\
\hline
\end{tabular}

Abbreviation: MA microalbuminuria, $B M I$ body mass index, SBP systolic blood pressure, DBP diastolic blood pressure, $H D L-c$ high-density lipoprotein-cholesterol, $L D L-c$ low-density lipoprotein-cholesterol, FBG fasting blood glucose, eGFR estimated glomerular filtration rate, UMA urinary albumin, UA uric acid, $h s C R P$ high-sensitivity Creactive protein, $N T$-proBNP N-terminal prohormone of brain natriuretic peptide, $A C E I / A R B$ angiotensin converting enzyme inhibitors/angiotensin receptor blockers

and proportions of cigarette smoking, hypertension and angiotensin converting enzyme inhibitor/angiotensin receptor blocker treatment in participants with MA significantly exceeded those in participants without MA.

\section{Single-marker analysis}

In the first and second steps of linear (Table 2) and logistic (Table 3) regression models, homocysteine and NT-proBNP levels $(p<0.05$ for all) rather than hsCRP and UA levels $(p>0.05$ for all) were statistically significant in relation to UMA or MA. Additionally, no matter which biomarker was directed at in the first and second steps, levels of age, SBP and FBG and proportion of cigarette smoking had significant associations with MA $(p<0.05$ for all).

\section{Multi-marker analysis}

In the third steps of linear (Table 2) and logistic (Table 3) regression models, homocysteine and NT-proBNP levels $(p<0.05$ for all $)$ rather than hsCRP and UA levels $(p>0.05$ for all) were statistically significant in relation to UMA or MA. Additionally, no matter which biomarker was directed at in the third steps, levels of age, SBP and FBG and proportion of cigarette smoking had significant associations with MA. Homocysteine and NT-proBNP levels $(p<0.05$ for all) rather than hsCRP and UA levels ( $p>0.05$ for all) had significant abilities to identify MA (Table 4 and Fig. 1).

\section{Discussion}

In this community population, we observed a significant relationships of homocysteine and NT-proBNP with MA even after multivariate adjustment. These data suggested that homocysteine and NT-proBNP representing different pathophysiologic pathways can promote the identification of individuals with increased risk for developing renal damage. In addition, these findings demonstrated several pathophysiologic pathways playing a potential role in the pathogenesis of renal damage.

Produced by the demethylation of methionine, homocysteine has been considered to have significant association with MA in some studies $[10,11]$, but not in other study [12]. This analysis extended the current literature and reported that increased homocysteine levels were independently associated with MA in Chinese community population [13]. The pathophysiologic pathways between hyperhomocysteinemia and MA remain unclear. Previous studies have suggested that homocysteine-related 
Table 2 Single-marker and multi-marker analyses in the linear regression

\begin{tabular}{|c|c|c|c|}
\hline \multirow[t]{2}{*}{ Biomarkers } & \multirow[t]{2}{*}{ Models } & \multicolumn{2}{|l|}{ UMA } \\
\hline & & Standardized $\beta$ value & $P$ value \\
\hline \multirow[t]{3}{*}{ Homocysteine $(\mu \mathrm{mol} / \mathrm{L})$} & 1st step ${ }^{a}$ & 0.275 & $<0.001$ \\
\hline & 2nd step ${ }^{b}$ & 0.236 & $<0.001$ \\
\hline & 3rd step ${ }^{c}$ & 0.223 & $<0.001$ \\
\hline \multirow[t]{3}{*}{ HsCRP (mg/dl) } & 1st step ${ }^{a}$ & -0.018 & 0.590 \\
\hline & 2nd step ${ }^{b}$ & -0.005 & 0.888 \\
\hline & 3rd step ${ }^{c}$ & -0.002 & 0.943 \\
\hline \multirow[t]{3}{*}{ NT-proBNP (pg/mL) } & 1st step ${ }^{a}$ & 0.152 & $<0.001$ \\
\hline & 2nd step ${ }^{b}$ & 0.140 & $<0.001$ \\
\hline & $3 r d$ step $^{c}$ & 0.129 & $<0.001$ \\
\hline \multirow[t]{3}{*}{ UA ( $\mu \mathrm{mol} / \mathrm{L})$} & 1st step ${ }^{a}$ & 0.001 & 0.987 \\
\hline & 2nd step ${ }^{b}$ & -0.049 & 0.199 \\
\hline & $3 r d$ step $^{c}$ & -0.054 & 0.148 \\
\hline
\end{tabular}

Note: ${ }^{a}$ First step: single biomarker in the linear regression model adjusted by age and gender; ${ }^{b}$ Second step: single biomarker in the linear regression model adjusted by age, gender, body mass index, cigarette smoking, systolic blood pressure, triglyceride, high-density lipoprotein-cholesterol, low-density lipoprotein-cholesterol, fasting blood glucose and glomerular filtration rate; 'Third step: all biomarkers simultaneously in the linear regression model adjusted by age, gender, body mass index, cigarette smoking, systolic blood pressure, triglyceride, high-density lipoprotein-cholesterol, low-density lipoprotein-cholesterol, fasting blood glucose and glomerular filtration rate Abbreviation: UMA urinary albumin, $h s C R P$ high-sensitivity C-reactive protein, NTproBNP N-terminal prohormone of brain natriuretic peptide, UA: uric acid

Table 3 Single-marker and multi-marker analyses in the logistic regression

\begin{tabular}{|c|c|c|c|}
\hline \multirow[t]{2}{*}{ Biomarkers } & \multirow[t]{2}{*}{ Models } & \multicolumn{2}{|l|}{ MA } \\
\hline & & OR value $(95 \% \mathrm{Cl})$ & $P$ value \\
\hline \multirow[t]{3}{*}{ Homocysteine $(\mu \mathrm{mol} / \mathrm{L})$} & 1st step ${ }^{a}$ & $1.066(1.041-1.092)$ & $<0.001$ \\
\hline & 2nd step ${ }^{b}$ & $1.059(1.031-1.087)$ & $<0.001$ \\
\hline & 3rd step ${ }^{c}$ & $1.059(1.032-1.087)$ & $<0.001$ \\
\hline \multirow[t]{3}{*}{ HsCRP (mg/dl) } & 1st step ${ }^{a}$ & $0.742(0.426-1.293)$ & 0.293 \\
\hline & 2nd step ${ }^{b}$ & $0.544(0.256-1.160)$ & 0.115 \\
\hline & $3 r d$ step $^{c}$ & $0.490(0.218-1.098)$ & 0.083 \\
\hline \multirow[t]{3}{*}{ NT-proBNP (pg/mL) } & 1st step ${ }^{a}$ & $1.002(1.001-1.003)$ & 0.006 \\
\hline & 2nd step ${ }^{b}$ & $1.002(1.000-1.003)$ & 0.020 \\
\hline & 3 rd step ${ }^{c}$ & $1.001(1.000-1.003)$ & 0.043 \\
\hline \multirow[t]{3}{*}{ UA $(\mu \mathrm{mol} / \mathrm{L})$} & 1st step ${ }^{a}$ & 1.002 (0.999-1.005) & 0.111 \\
\hline & 2nd step ${ }^{b}$ & $1.001(0.998-1.005)$ & 0.415 \\
\hline & $3 r d$ step $^{c}$ & $1.001(0.998-1.005)$ & 0.478 \\
\hline
\end{tabular}

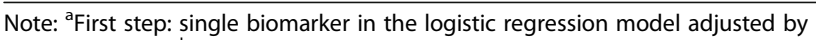
age and gender; ${ }^{b}$ Second step: single biomarker in the logistic regression model adjusted by age, gender, body mass index, cigarette smoking, systolic blood pressure, triglyceride, high-density lipoprotein-cholesterol, low-density lipoprotein-cholesterol, fasting blood glucose and glomerular filtration rate; 'Third step: all biomarkers simultaneously in the logistic regression model adjusted by age, gender, body mass index, cigarette smoking, systolic blood pressure, triglyceride, high-density lipoprotein-cholesterol, low-density lipoprotein-cholesterol, fasting blood glucose and glomerular filtration rate Abbreviation: MA microalbuminuria, OR odd ratio, $C l$ confidence interval, $h s C R P$ high-sensitivity C-reactive protein, NT-proBNP N-terminal prohormone of brain natriuretic peptide, $U A$ uric acid
Table 4 Abilities of biomarkers to identify MA

\begin{tabular}{lll}
\hline & AUC value $(95 \% \mathrm{Cl})$ & $P$ value \\
\hline Homocysteine $(\mu \mathrm{mol} / \mathrm{L})$ & $0.627(0.572-0.682)$ & $<0.001$ \\
HsCRP $(\mathrm{mg} / \mathrm{dl})$ & $0.453(0.393-0.513)$ & 0.108 \\
NT-proBNP $(\mathrm{pg} / \mathrm{mL})$ & $0.636(0.585-0.687)$ & $<0.001$ \\
UA $(\mu \mathrm{mol} / \mathrm{L})$ & $0.522(0.465-0.579)$ & 0.455 \\
\hline
\end{tabular}

Abbreviation: $M A$ microalbuminuria, $A U C$ area under the curve, $\mathrm{Cl}$ confidence interval, $h s C R P$ high-sensitivity C-reactive protein, NT-proBNP N-terminal prohormone of brain natriuretic peptide, UA uric acid

oxidative stress injures endothelial and mesangial cells [14]. Renal endothelial and mesangial cells are significant for not only controlling intraglomerular pressure, but also preserving glomerular size and charge selectivity. If they are injured, there will be elevated intraglomerular pressure, and reduced glomerular charge and size selectivity, both causing MA. Additionally, there are other common pathophysiologic pathways, such as vitamin B6, B12 and folate deficiency, resulting in both hyperhomocysteinemia and MA [15].

Meanwhile, this analysis identified NT-proBNP as a biomarker associated with MA. Previous study has suggested that NT-proBNP have significant association with renal damage [8]. Because NT-proBNP is removed by kidney, early reduction of NT-proBNP clearance can account for our finding [16]. Besides, natriuretic peptides can prevent glomerular hyperfiltration, fibrosis and hypertrophy in previous experiments, so NT-proBNP may have protective effect on kidney [17-19].

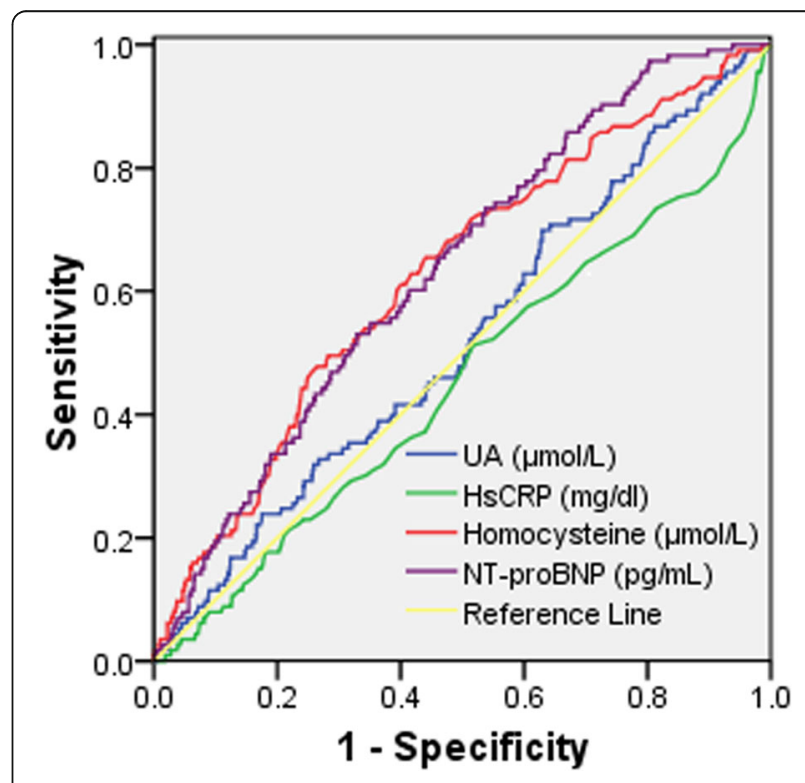

Fig. 1 Receiver Operator Characteristic Curve for abilities of biomarkers to identify MA. Abbreviation: hsCRP high-sensitivity C-reactive protein, NT-proBNP N-terminal prohormone of brain natriuretic peptide, UA uric acid 
The current analysis had strength and limitations. The current analysis specially aimed at Chinese middle-aged and elderly from communities, and had a large number of participants without missing values for any covariates. Moreover, it clarified the associations between biomarkers and MA through constructing single-marker and multimarker models of linear and logistic regression analyses simultaneously. But UMA was evaluated on at least $5 \mathrm{~mL}$ of midstream urine from morning urine rather than 24-h urine. It is difficult and unpractical to measure 24-h urine in epidemiological investigations.

\section{Conclusion}

Both single-marker and multi-marker analyses provided proof that homocysteine and NT-proBNP levels were significantly associated with MA in Chinese middle-aged and elderly from communities after adjustment for multiple confounders. These biomarkers representing different pathophysiologic pathways can enhance the identification of individuals with increased risk for developing renal damage. More importantly, these findings can help us understand these potential pathways involved in the pathogenesis of renal damage.

\section{Acknowledgments}

We thank all participants for their help.

\section{Funding}

The current analysis was funded by National Key Basic Research Program (2012CB517503 and 2013CB530804), Health Special Scientific Research Program of Chinese People's Liberation Army (12BJZ34 and 14BJZ12), and Sanya Medical and Health Science and Technology Innovation Program (2016YW21).

Availability of data and materials

Information of patients can not be shared so as to preserve the privacy; data are available upon request.

\section{Authors' contributions}

Study design: FS, GY, ZZ, LLM, YP; data collection and analysis: FS, GY, ZZ, LLM, YP; paper draft: FS, GY, ZZ, LLM, YP. All authors read and approved the final manuscript.

\section{Ethics approval and consent to participate}

Ethics Committee of Chinese People's Liberation Army General Hospital approved the study, with written consents obtained.

\section{Competing interests}

The authors declare that they have no competing interests.

\section{Publisher's Note}

Springer Nature remains neutral with regard to jurisdictional claims in published maps and institutional affiliations.

\section{Author details}

'Department of Geriatric Cardiology, Chinese People's Liberation Army General Hospital, Beijing, China. ${ }^{2}$ Department of Cardiology and Hainan Branch, Chinese People's Liberation Army General Hospital, Beijing, China.
Received: 25 November 2016 Accepted: 5 April 2018

Published online: 23 April 2018

\section{References}

1. Singh M. The cardiorenal connection: new insights toward choice of revascularization. J Am Coll Cardiol. 2014;64:995-6.

2. House AA. Cardiorenal syndrome: introduction. Clin J Am Soc Nephrol. 2013:8:1798-9.

3. Lawrence J, Bai S, Hung HM, O'Neill R. Regional treatment effects in studies of cardiorenal drugs: a summary of recent clinical trials. J Am Coll Cardiol. 2012;60:1117-8

4. Bakris GL. Slowing nephropathy progression: focus on proteinuria reduction Clin J Am Soc Nephrol. 2008:3:S3-S10.

5. Fox CS, Gona P, Larson MG, et al. A multi-marker approach to predict incident CKD and microalbuminuria. J Am Soc Nephrol. 2010:21:2143-9.

6. Resl M, Clodi M, Neuhold S, et al. Serum uric acid is related to cardiovascular events and correlates with $\mathrm{N}$-terminal pro-Btype natriuretic peptide and albuminuria in patients with diabetes mellitus. Diabet Med. 2012:29:721-5.

7. de Jong PE, Curhan GC. Screening, monitoring, and treatment of albuminuria: Public health perspectives. J Am Soc Nephrol. 2006;17:2120-6.

8. Yi S, Contreras G, Miller ER, Appel LJ, Astor BC. Correlates of Nterminal prohormone brain natriuretic peptides in African Americans with hypertensive chronic kidney disease: the African American study of kidney disease and hypertension. Am J Nephrol. 2009;29:292-8.

9. Ma YC, Zuo L, Chen JH, et al. Modified glomerular filtration rate estimating equation for Chinese patients with chronic kidney disease. J Am Soc Nephrol. 2006;17:2937-44.

10. Hoogeveen EK, Kostense PJ, Jager A, et al. Serum homocysteine level and protein intake are related to risk of microalbuminuria: the Hoorn study. Kidney Int. 1998;54:203-9.

11. Sabanayagam C, Shankar A. Association between plasma homocysteine and microalbuminuria in persons without hypertension, diabetes mellitus and cardiovascular disease. Clin Exp Nephrol. 2011;15:92-9.

12. Li J, Shi M, Zhang H, et al. Relation of homocysteine to early nephropathy in patients with type 2 diabetes. Clin Nephrol. 2012;77:305-10.

13. Ninomiya T, Kiyohara $Y$, Kubo $M$, et al. Hyperhomocysteinemia and the development of chronic kidney disease in a general population: the Hisayama study. Am J Kidney Dis. 2004:44:437-45.

14. Tsai JC, Perrella MA, Yoshizumi M, et al. Promotion of vascular smooth muscle cell growth by homocysteine: a link to atherosclerosis. Proc Natl Acad Sci U S A. 1994:91:6369-73.

15. Miliku K, Mesu A, Franco OH, et al. Maternal and fetal folate, vitamin B12, and homocysteine concentrations and childhood kidney outcomes. Am J Kidney Dis. 2017;69:521-30

16. van Kimmenade RR, Januzzi JL Jr, Bakker JA, et al. Renal clearance of B-type natriuretic peptide and amino terminal pro-B-type natriuretic peptide: $A$ mechanistic study in hypertensive subjects. J Am Coll Cardiol. 2009;53:884-90.

17. Suganami T, Mukoyama M, Sugawara A, et al. Overexpression of brain natriuretic peptide in mice ameliorates immune-mediated renal injury. J Am Soc Nephrol. 2001;12:2652-63.

18. Makino H, Mukoyama M, Mori K, et al. Transgenic overexpression of brain natriuretic peptide prevents the progression of diabetic nephropathy in mice. Diabetologia. 2006;49:2514-24.

19. Dieplinger B, Mueller T, Kollerits B, et al. Pro-A-type natriuretic peptide and pro-adrenomedullin predict progression of chronic kidney disease: the MMKD study. Kidney Int. 2009:75:408-14. 